\title{
SISTEMA DE COSTOS EN MYPES INDUSTRIALES Y DE SERVICIOS EN CONDICIONES DE DESORGANIZACIÓN
}

\author{
DISORGANIZED TERMS FROM MYPES WITH A SYSTEM COSTS \\ AND SERVICES INDUSTRIAL \\ JoRge Jesús GAVElán IzAgUiRRE* \\ Docente Asociado de la Facultad de Ciencias Contables \\ Universidad Nacional Mayor de San Marcos-UNMSM / Lima-Perú \\ [Recepción: Marzo de 2014/ Conformidad: Mayo 2014]
}

\section{RESUMEN}

En el Perú, existen limitaciones para formular y aplicar un Sistema de Costos convencional, a través del cual se identifique los elementos del costo de producción atribuibles a un lote de productos elaborados o servicios prestados, debido a que en las empresas no se aplica regular y permanente los principios generales de la administración, en particular: Organización y Control, a lo que suma el alto costo de la formalidad laboral y tributaria.

Cambiar la mentalidad de los directivos de las MYPES para crear las condiciones que permita establecer un óptimo sistema de costos, es una labor persistente y de lento efecto. La única manera es que estos perciben su utilidad efectiva y contribuyen a la generación de la rentabilidad selectiva por productos elaborados o clase de servicios, así como a optimizar en la administración de los recursos.

El reto para el Contador Público es administrar la desorganización, organizando la acumulación, distribución y aplicación del costo de producción, en función de las condiciones existentes en la empresa, dejado parcialmente de lado, los procedimientos convencionales de costeo, y suplir "los vacíos" con otros medios aplicativos.

\section{Palabras clave:}

Sistemas de costos; elementos del costo de producción; costo real; costo estimado; MYPES.

\begin{abstract}
In Peru, there are limitations to develop and implement a conventional system costs, through which the elements of production costs attributable to a lot of processed products or services are identified, because in companies is not applied regularly and permanent general management principles, including: Organization and Control, which adds to the high cost of labor and tax formalities.

Changing the mindset of managers of MSEs to create the conditions to establish optimal cost system is a slow-acting and persistent work. The only way is that they perceive their actual utility and contribute to the generation of selective yield processed products or class of services and to optimize the management of resources.

The challenge for the public accountant is to administer the disorganization by organizing the buildup, with distribution and application of production cost, depending on the conditions in the company, partly left side, conventional costing procedures and fill "gaps" with other media applications.
\end{abstract}

\section{Keywords:}

Cost systems; elements of cost of production; actual cost; estimated cost; MSEs.

* Contador Público Colegiado. UNMSM. Email: jjgavelani@yahoo.es 


\section{INTRODUCCIÓN}

El presente artículo expone inquietudes propias respecto a la formulación y aplicación de un sistema de costos en las Medianas y Pequeñas Empresas (MYPES) industriales y de servicios, observando frecuentemente que en estas no hay condiciones óptimas para su desarrollo y aplicación, dado que existe hábitos negativos tan arraigados que gira sobre el de no aplicar a plenitud los principios de la administración: Planeación, organización, dirección y control. Este artículo pretende orientar al Contador Público cómo formular y desarrollar un sistema de costos al margen de los tratamientos convencionales, adaptados a empresas que en un primer momento no son proclives a optimizar los procesos y prácticas de trabajo relacionados con la obtención de los costos de producción.

El artículo se encuentra enmarcado en lo que podría denominarse una ciencia empírica y personal, creada y alimentada por la experiencia y sobretodo, orientada a alcanzar el objetivo de diseñar y aplicar un sistema de costos adaptada a una empresa que opera en condiciones de desorganización y descontrol, y que por lo menos, transitoriamente, no está apta para cambiar y optimizar íntegramente sus procesos, salvo lo que la gerencia considera estrictamente indispensable. Este tratamiento de costeo frecuentemente y sobretodo en su fase inicial, gira sobre una visión general de la empresa y, en particular, de las áreas comprometidas o a comprometer, no es categórico y riguroso, porque consiste en un ensayo reflexivo, libre de ataduras metodológicas y flexible que descansa sobre la base de la aplicación, error, corrección y aplicación (error corregido).

En el Perú, la actividad económica a través de las empresas es abrumadoramente informal, que se calcula en un 65\% en comparación a las comprendidas en la formalidad (35\%), lo que implica que en estas no se aplican las leyes establecidas, en particular las de carácter laboral y tributario ¿Por qué el empresario peruano, sobre todo a nivel de MYPES opta por operar al margen de la ley? Nos preguntamos iserá el efecto de una mala educación, carente de valores? o ¿será inherente y congénito a los peruanos? Esta última respuesta sería doblemente preocupante. Sin embargo, para las personas meridianamente conocedoras del tema, tienen una respuesta: Nuestros congresistas redactores y emisores de leyes, así como, las entidades públicas encargadas de su reglamentación, aplicación y fiscalización operan en un "mundo ideal" ajeno al "Perú real", dado que las formalidades, procedimientos y cargas fiscales son exageradas, frecuentemente en conflicto con la racionalidad y que constantemente sacrifican al "criterio común" en aras de proceder de acuerdo al "mundo ideal", haciendo muy oneroso su cumplimiento, restando rentabilidad y eficiencia a las empresas peruanas.

La situación enunciada ha sido comentada por el diario (ElComercio, 2014) en su editorial de fecha 06 de abril, titulada "Historia de dos países" (en alusión al Perú legal y al Perú real) y que considero necesario para los fines del presente, reproducir algunos párrafos:

"En cuanto al aspecto tributario, es ilustrativo decir que nuestro impuesto a la renta empresarial está casi 10 puntos porcentuales por encima del promedio de los 34 países más desarrollados del mundo (agrupados en la Organización para la Cooperación y Desarrollo Económicos-OCDE)". "En lo que toca a las barreras burocráticas a los negocios en general, finalmente, ocupamos el puesto 113 de 139 países en la categoría de "peso de las regulaciones burocráticas" del Reporte Global de Competitividad. Peor aún, estamos bastante por detrás (es decir, tenemos bastante más exigentes regulaciones) que el antes referido promedio de los países de la OCDE, lo que es particularmente significativo, si tomamos en cuenta que no tenemos ni de cerca el nivel de protección para los diferentes derechos de terceros que hay en estos países".

Tome nota el lector de las difíciles condiciones de regulación legal en que debe operar las empresas peruanas, lo que incide significativamente en la decisión para implementar un sistema de costos en una empresa. Lo primero que se pregunta el Contador es si existe las condiciones para su formulación y aprobación como: organización, disciplina y controles internos y, si efectivamente, la gerencia está firmemente convencida de su impostergable implementación; caso contrario, estos pueden quedar en "buenas intenciones" y "en el papel” y la labor desplegada en su 
desarrollo se convierte en una pérdida de recursos y "desgaste de personal" en una obra infructuosa, lo que tiene un efecto desmoralizador en la organización.

\section{FUNCIÓN DEL CONTADOR PÚBLICO}

La Contabilidad de Costos de Servicios e Industrial es parte de la contabilidad general que a través de registros y cuentas especiales, recopila información, clasifica, distribuye, acumula y controla el uso de recursos humanos y materiales, asignando los costos a las actividades conjuntas efectuadas en la ejecución de una prestación requerida por terceras personas, así como, emitir información para el control de las operaciones realizadas.

Un sistema de contabilidad de costos de servicios (o industriales) está relacionado con la actividad que realiza la empresa y como tal, existe la necesidad de establecer un modelo, en el que no solo se incorpore los elementos que constituyen el costo, sino que además, se determine cómo incorporar los costos reales y los predeterminados y un sistema de costos por procesos o un sistema de costos por órdenes de fabricación o costeo por actividades. El sistema de costos de servicios está más asociado con un sistema de costos por órdenes de fabricación y a costos predeterminados $y$ esto, se debe a que generalmente cada servicio prestado al cliente es distinto de otro, pero si hubiera similitud en la prestación de un grupo de servicios, es susceptible reunir estos y costear en forma agrupada, en función al número de veces que da el servicio en el mes, por ejemplo el caso de intervenciones quirúrgicas por cesárea en el mes.

Todo sistema de costos tiene objetivos a alcanzar, resumiéndolos, tenemos:

a. Determinar el costo acumulado y unitario por cada servicio, lote o proceso ejecutado.

b. Controlar el costo de producción ejecutado comparándolo con el presupuesto a efectos de identificar las desviaciones, evaluando de esta forma la eficiencia operativa de la empresa.

c. Facilitar la toma de decisiones para el planeamiento de servicios requeridos (o bienes a producir), así como, para definir políticas de venta y fijación de precios en función a cada clase de servicios o productos.
Cabe agregar, que para cumplir los objetivos señalados previamente es indispensable conocer las actividades que realiza la empresa, así como, los procedimientos y procesos que se ejecutan para brindar el servicio o producir bienes. Este conocimiento es el punto de partida que apropiadamente interpretado, es la base para determinar las características del sistema de costos a diseñar y aplicar.

¿Será posible alcanzar los objetivos señalados, considerando que en el Perú la mayoría de las MYPES industriales y de servicios carecen de un sistema de contabilidad de costos? En los casos en que se determina los costos, estos son formulados al margen de los libros de contabilidad. La formulación de los costos frecuentemente está a cargo del área operativa que presta servicios a los clientes o que tiene a su cargo el proceso de fabricación, generalmente, a cargo de Ingenieros Industriales o de otras especialidades de la ingeniería, pero no efectúa "cruce" riguroso con la contabilidad, dado que el área contable registra y genera información (real) acumulada de costos, no identificada por actividad específica o servicio brindado al cliente. ¿Por qué se produce esta situación? La respuesta está en que cada área "camina por su lado", no se integra la labor de costos.

¿El área de contabilidad ha de registrar y asumir responsabilidad por los costos formulados por otra área a cargo de profesionales que no son Contadores Públicos, más aún si las sumas no son coincidentes con los costos reales acumulados en la cuenta 92 Costo de producción o 93 Centros de costos, no identificados con la actividad productiva específica? La respuesta es sí, previa coordinación y verificación de la información obtenida, a cargo de Contadores Públicos. El objetivo es optimizar y racionalizar el trabajo de costeo, evitando la duplicidad de trabajo. Es contraproducente actuar en función de celos profesionales, que devienen muchas veces en absurdos y hasta risibles, que implica inseguridad y baja autoestima. Es mucho mejor coordinar el trabajo de costeo a realizar, verificar y corregir; que realizar el mismo trabajo dos veces y en forma diferente.

En las organizaciones deben haber tres cualidades básicas para implementar un sistema de costos: Organización, control y disciplina $i$ Se encuentran estas 
presentes en el común de las empresas peruanas?. Si la respuesta fuera NO, entonces las MYPES están "condenadas" a no tener un sistema de costos, dado que es "vox populi" que las cualidades señaladas no abundan en estas. ¿Qué hacer? Respuesta: desarrollar e implementar el sistema de costos de acuerdo a las condiciones existentes y posibilidades mínimas de mejoras organizativas reales, hasta que la gerencia perciba las ventajas de tener un sistema de costos, inicialmente "aproximado", pero luego progresivamente real.

\section{ELEMENTOS DEL COSTO DEL SERVICIO}

El costo de producción de servicios (aplicable también a las industrias a través de la cuenta $24 \mathrm{Ma}$ terias Primas) se obtendrá por acumulación de las cuentas de costos, clasificado en sus tres elementos: Materiales directos, mano de obra directa y gastos indirectos de producción.

Material directo.- Son los insumos objeto de conversión en productos elaborados o suministros utilizados en la prestación de servicios. Este elemento se incorpora al costo cuando se ha emitido una "Guía de Salida" por el Almacén, por el cual se ha retirado el material para su consumo o uso en el área de producción o de prestación de servicios, valorizándose de acuerdo al costo unitario de adquisición obtenido del "Kardex valorizado de Almacén" (o su equivalente computarizado), formulando, además, el asiento de diario por la salida del Almacén.

613 Materiales auxiliares, suministros y repuestos

252 a Suministros

921 Costo de producción - Materiales

791 a Cargas imputables a cuentas de costos
Cuando las empresas no operan de acuerdo al tratamiento regular antes señalado, debido a que carecen de un sistema de costos o su aplicación es incompleta e irregular y en el ánimo de implementar un sistema de costos menos riguroso, pero razonable, podría costearse los materiales bajo las siguientes alternativas:

Caso $\mathrm{N}^{\circ} 1$. No existe kardex valorizado de materiales, pero sí kardex físico. En este caso, siempre que exista la “Guía de Salida”, se procede a valorizar por cada tipo de material directamente de la factura de compra, habiendo hasta tres modalidades:

1.1. Valorizar las salidas en base a la última compra, lo que implica en los hechos aplicar el método de valuación de existencias UEPS.

1.2. Valorizar las salidas en base a la compra anterior a la última, siempre que no se identifique la compra específica, o que tiene por efecto aplicar indirectamente (y por aproximación) el método de valuación de existencias PEPS.

1.3. Valorizar las salidas en base al costo promedio ponderado de adquisición de las dos últimas compras. Las existencias finales se valorizan con el último costo de adquisición.

A fin de mes y sustentado en una hoja de trabajo de valorización de existencias, se formula el asiento contable de diario por la salida del Almacén; así por ejemplo:

$$
\begin{array}{rr}
\text { S/. } 180400 & \text { S/. } \\
180400 & 180400 \\
& 180400
\end{array}
$$

Luego, en el Libro Mayor al registrar el consumo de los suministros utilizados en la prestación de servicios por s/. 180400 en la subcuenta 252 Suministros, aparece entre otros, la siguiente información:

\begin{tabular}{lr|lr} 
DEBE & \multicolumn{2}{l}{ 252 Suministros } & HABER \\
\hline Saldo inicial & S/.31 000 & Consumo & S/. 180400 \\
Compras & 242200 & & \\
Sumas & 273200 & Sumas & 180400 \\
& & Saldo final (preliminar) & 92800
\end{tabular}


El saldo final del Libro Mayor, debe ser coincidente con la suma del inventario físico valorizado al final del período; pero es más posible que no coincidan por efecto de la valorización (no por sobrantes o faltantes de inventario). Sin embargo, el inventario físico valorizado al último costo de adquisición de cada artículo, reemplaza al saldo final (preliminar) de

\section{Suministros}

613 a Materiales auxiliares, suministros y repuestos

791 Cargas imputables a cuentas de costos

921 a Costo de producción - Materiales la subcuenta 252; así por ejemplo: Al 31-12-2013 el saldo final de la subcuenta 252 Suministros es S/. 92 800 , y según el inventario físico es S/.94 100, luego el saldo final (preliminar) se muestra menor, debido a que se ha registrado el costo de producción por una suma mayor en S/. 1300, luego se procede a formular el A/D de ajuste del costo de producción:

$$
\begin{array}{rr}
\text { S/. } 1300 & \text { S/. } \\
1300 & 1300 \\
& 1300
\end{array}
$$

Luego, al registrar en el Libro Mayor, subcuenta 252 Suministros, el A/D en mención, mostrará:

DEBE

Saldo inicial

Compras

Ajuste de costo

Sumas
252 Suministros

HABER

S/. 31000

242200

1300

273200
Consumo

Sumas

Saldo final (preliminar)
S/. 180400

180400

94100

\section{Caso $\mathbf{N}^{\circ}$ 2. No existe kardex valorizado de ma-} teriales, ni kardex físico, pero síla "Guía de salida”. En este caso, proceder a valorizar el documento de salida de materiales directamente de la factura de compra, generando además, el asiento contable de diario por la salida del Almacén. Pudiéndose aplicar alguna de las tres alternativas:

1.1. Valorizar las salidas en base a la última compra, lo que implica en los hechos aplicar el método de valuación de existencias UEPS.

1.2. Valorizar las salidas en base a la compra anterior a la última, siempre que no sea posible identificar la compra específica, lo que tiene por efecto aplicar indirectamente (y por aproximación) el método de valuación de existencias PEPS.

1.3. Valorizar las salidas en base al costo promedio ponderado de adquisición de las dos últimas compras. Las existencias finales se valorizan con el último costo de adquisición.

Dado que no existe ningún registro para anotar y controlar el movimiento de entradas y salidas de almacén, la suma consumida registrada como costo de producción del período, carece de un registro de soporte y el saldo final de la subcuenta $252 \mathrm{Su}$ ministros no tiene detalle analítico y sustentación, es imprescindible formular el inventario físico, por lo menos al final del período y proceder a su valorización, luego en el Libro Mayor, la subcuenta 252 Suministros, se "cruza" contra el inventario físico valorizado, por la diferencia entre las sumas, se efectúa el ajuste correspondiente; así por ejemplo, si en el Libro Mayor al cierre de libros (preliminar), se hubiera registrado lo siguiente:
DEBE

Saldo inicial

Compras

Sumas
252 Suministros

HABER

\begin{tabular}{r|lr} 
S/. 36000 & Consumo & S/. 190200 \\
288200 & & 190200 \\
324200 & Sumas & 134000
\end{tabular}


El inventario físico valorizado de suministros al 31-12-2013 es S/. 128 200, luego hay un exceso de saldo final preliminar de S/. 5 800, lo que significa que se ha registrado costos de menos, debiéndose formular el siguiente A/D:

613 Materiales auxiliares, suministros y repuestos

252 a Suministros

921 Costo de producción - Materiales

$\begin{array}{rr}\text { S/. } 5800 \quad \text { S/. } \\ 5800 & 5800 \\ & 5800\end{array}$

791 a Cargas imputables a cuentas de costos

Al proceder a la mayorización del ajuste en la subcuenta 252 Suministros, ha de coincidir el saldo final de libros con el inventario físico, según se observa a continuación:

DEBE 252 Suministros

HABER

Saldo inicial

Compras

S/. 36000

Consumo

S/. 190200

Sumas

288200

Ajuste de costo

5800

$\begin{array}{lr}\text { Sumas } & 196000\end{array}$

Saldo final (preliminar)

128200

Caso $\mathbf{N}^{\circ}$ 3. No existe kardex valorizado de materiales, ni kardex físico, ni la "Guía de salida".

Este caso es una situación extrema, se carece de un documento que nos permita registrar el costo de los materiales empleados en la fabricación de un producto o prestación de servicios, luego estamos imposibilitados de incorporar el costo, salvo la posibilidad que la empresa tenga por práctica regular formular "Presupuestos" previos de atención a cada cliente, y que estos estén formulados analíticamente y con rigurosidad, y que ejerzan control efectivo (extracontable) de su aplicación. En este caso, se registrará contablemente como costo de los materiales el valor presupuestal señalado, suma sujeta a reajuste, cuando se formule el inventario físico valorizado de fin de año.

En esta situación el costo de los materiales consumidos es a valor estimado, debiendo al final del período convertir a un costo real, para lo cual es imprescindible formular el inventario físico y proceder a su valorización, suma que al cierre de libros ha de corregir el saldo preliminar de la subcuenta 252 Suministros; así por ejemplo, el Libro Mayor de la subcuenta en mención, muestra lo siguiente (preliminar):
DEBE

Saldo inicial

Compras

Sumas
252 Suministros

\begin{tabular}{r|l} 
S/. 41500 & Consumo \\
287100 & \\
328600 & Sumas \\
& Saldo final (preliminar)
\end{tabular}

HABER

S/. 210000

210000 118600

El inventario físico valorizado a costo real al 31-12-2013 es s/. 114 800, luego el saldo final está sobrevaluado en s/. 3800 y para convertir la cuenta 252 a saldo real, y por extensión el costo de producción, se ha de formular el siguiente $A / D$ :

613 Materiales auxiliares, suministros y repuestos

252 a Suministros

S/. 3800

S/.

921 Costo de producción - Materiales

3800

3800

791 a Cargas imputables a cuentas de costos

3800

El efecto de registrar el A/D en el libro mayor por la cuenta 252, es el siguiente:

DEBE

Saldo inicial (Costo real)

Compras

Sumas
252 Suministros

HABER

\begin{tabular}{r|lr} 
S/. 41500 & Consumo (a costo estimado) & S/.210 000 \\
287100 & Ajuste de costo & 3800 \\
328600 & Sumas & 213800 \\
& Saldo final (preliminar) & 114800
\end{tabular}

126/ QVIPUIRAMAYOC | Vol. 22(41) 2014 
Como se observa en el Libro Mayor, al final del período se refleja el saldo real, sustentado en el inventario físico valorizado.

Mano de Obra Directa. Está constituido por el costo de los servicios de los trabajadores que laboran directamente en la prestación del servicio del área a que corresponden o en la elaboración de los bienes previstos. Dado que las características operativas de las empresas de prestación de servicios, tienen mayor similitud con las empresas industriales que aplican un sistema de costos por Órdenes de Producción, es necesario diferenciar las sumas registradas en la planilla de remuneraciones, de las que constituye el uso efectivo o real del trabajo, dado que no siempre las sumas pagadas es equivalente al empleo total de la fuerza laboral en producción (existe en la empresa trabajadores en exceso).

Cuando la empresa no ejerce control del uso efectivo de la Mano de Obra, y que se persista en implementar un sistema de costos menos exacto, pero razonable, podría costearse bajo las siguientes alternativas:

Caso $\mathbf{N}^{\circ}$ 1. No existe informe de las horas-hombre utilizadas, pero si el informe del supervisor de campo, del cumplimiento de labores. En este caso se costeará la mano de obra en función al número de trabajadores que han intervenido en la prestación del servicio (o fabricación de bienes), multiplicado por el valor promedio del jornal del trabajador, el total se registra en el mes al costo de producción. Después del cierre contable del mes, se cruzará estas sumas contra el valor de la planilla de remuneraciones, por la diferencia de costos, se formulará un asiento contable, incrementando o disminuyendo el costo. Las contribuciones y cargas sociales serán asignadas adicionalmente, formulando coeficientes, sujeto a posterior ajuste del valor real.

Caso $\mathbf{N}^{\circ}$ 2. No existe informe de las horas-hombre utilizadas, ni informe de supervisor de campo. En este caso, dado que se carece de un documento que nos permita registrar el costo de la Mano de Obra empleada en la prestación de servicios (o fabricación de bienes), estamos imposibilitados de incorporar el costo, salvo la posibilidad que la empresa tenga por práctica regular formular "Presupuestos" previos de atención a cada cliente, y que estos estén formulados analíticamente y sean rigurosos en el cálculo y que se ejerza control efectivo (extracontable) de su aplicación. En este caso se registrará contablemente como costo de la Mano de Obra el valor presupuestado, suma sujeta a reajuste a valor real, cuando se cruce información con el total de la planilla de remuneraciones. El tratamiento de las contribuciones y cargos sociales, es igual al señalado en el párrafo anterior.

Gastos Indirectos. Son aquellos gastos no aplicados directamente al costo del servicio (o fabricación de bienes), pero necesario para dar la prestación y mantener la operatividad de la empresa. Los gastos indirectos de servicios comprenden tres categorías:

a. Material indirecto. Son aquellos que no se convierten en parte del servicio o del producto elaborado o que siéndolo su importancia es secundaria, como: Combustible, suministros de mantenimiento, reparación y limpieza, etc.

b. Mano de obra indirecta. Constituído por el personal que no trabaja físicamente en la prestación del servicio o en la elaboración del producto, sino en la dirección, supervisión e inspección general del proceso del servicio, mantenimiento, etc.

c. Gastos indirectos generales. Incluye recursos y servicios en general, conexos a la prestación del servicio o elaboración del producto, como: energía eléctrica, agua, alquiler, seguros contra incendios y siniestros en general, seguro de accidentes, depreciación de maquinaria, equipos e inmuebles, gastos de conservación y mantenimiento del edificio y equipos, impuestos y arbitrios municipales, etc.

No obstante, que reviste cierta dificultad la asignación de los gastos indirectos de servicios al costo, una vez adoptado un método, este debe efectuarse a través de una base sistemática y permanente de asignación o prorrateo; salvo que de un mes a otro se determine significativas diferencias con los costos indirectos reales, entonces será necesario cambiar el método por otro, cuyo efecto sea la mayor similitud entre el costo indirecto asignado y el costo real. Este último, debe identificarse en el Libro Mayor en la cuenta 92 Costo de producción o 93 Costo de producción, suma generalmente obtenida del lado del Debe del mayor. 
Determinar y ejercer un control del costo de producción a través de los tres elementos señalados, para la actividad industrial y de servicios es muy necesario a los directivos de la empresa, 'también es un requerimiento de las Normas Internacionales de Contabilidad (NIC's); así la NIC 2 Existencias, regula la formulación de los costos de servicios, en el párrafo 19 Coste de las existencias para un prestador de servicios, enuncia: "En el caso que un prestador de servicios tenga existencias, las valorará por los costes que suponga su producción. Estos costes se componen fundamentalmente de mano de obra y otros costes del personal directamente involucrado en la prestación del servicio, incluyendo personal de supervisión y otros costes indirectos distribuibles. La mano de obra y los demás costes relacionados con las ventas, y con el personal de administración general, no se incluirán en el coste de las existencias, pero se contabilizarán como gastos del ejercicio en el que se hayan incurrido. Los costes de las existencias de un prestador de servicios no incluirán márgenes de ganancia ni costes indirectos no distribuibles que, a menudo, se tienen en cuenta en los precios facturados por el prestador de servicios".

\section{MÉTODOS PARA ASIGNAR LOS GASTOS INDIRECTOS DE SERVICIOS A LA PRODUCCIÓN}

En los hechos, es materialmente imposible imputar a cada orden de servicio, los gastos indirectos de servicios (GIS) a valor real, por ser imposible determinar la cuantía exacta previa a su aplicación, dado que se produce un desfase cronológico del registro de gastos atribuible al costo y acumulación respectiva en las cuentas 91 Costos por distribuir ó 93 Centros de costo que se registran en cualquier día del mes; sin embargo, cuando la empresa aplica el sistema de costos por órdenes de fabricación, generalmente requiere atribuir una alícuota de gastos indirectos a las órdenes de fabricación o de servicios terminadas o en proceso antes del cierre de libros del mes, $y$ en ese momento no se cuenta con esa suma real por lo que existe necesidad de establecer una base de prorrateo que asigne los costos indirectos a cada servicio prestado a los clientes, mientras contablemente se acumulan en una cuenta de control, para posteriormente distribuirlos. Los métodos para prorratear los gastos indirectos a la producción son variados, pero se debe adoptar el que tenga por efecto obtener la base más equitativa de prorrateo, para lo cual se determina el elemento del costo directo del servicio (materiales o mano de obra) que sea el más importante por su incidencia o cuantía.

El método de prorrateo de los gastos indirectos más apropiados para las empresas prestadoras de servicios es el Prorrateo sobre la base de la Mano de Obra Directa, dado que generalmente es el más relevante en comparación a los otros dos elementos del costo. Este método se divide en dos: Base del valor y Base del tiempo; describiéndolos:

Base del Valor. Este método es apropiado cuando se presta un solo tipo de servicio; y si es más de uno, cuando las remuneraciones son iguales o semejantes en las secciones operativas de prestación de servicios. Consiste en dividir los gastos indirectos (GI) del mes o del ejercicio anterior entre el costo de la Mano de Obra Directa de igual período. Luego, el coeficiente del GI se multiplica por el costo de la Mano de Obra Directa atribuible a la orden de servicio, dando por resultado el importe del GI predeterminado. Posteriormente, se suma a los costos directos reales para obtener el costo de producción, que viene a ser una mezcla de valores reales y estimados, por consiguiente, periódicamente se ajusta la suma a valores reales; ejemplo:

La empresa Servicios Especializados S.A.C. requiere cuantificar el GI del mes de enero, para lo cual cuenta con el costo real del período anterior, constituido por:

$$
\begin{array}{lr}
\text { - Materiales } & \text { S/. } 2000000 \\
\text { - Mano de obra }(500000 \mathrm{H}-\mathrm{H}) & 2400000 \\
\text { - Gastos indirectos } & 1800000 \\
\text { COSTO DE PRODUCCIÓN (real) } & \mathbf{6 2 0 0 0 0 0}
\end{array}
$$

$$
\text { Coeficiente GIS }=\frac{1800000}{2400000}=0.75
$$

Si el coeficiente se aplica a la Orden de Servicio \# 001 del cliente Naylamp S.R.L., cuyos costo directos son: Materiales y Mano de obra (30 $000 \mathrm{H}-\mathrm{H})$ por S/. 200000 y S/. 300000 respectivamente, se obtendría: 
- Materiales

S/. 100000

- Mano de obra (30 $000 \mathrm{H}-\mathrm{H})$

150000

- Gastos indirectos de servicios:

$$
\mathrm{S} / .150000 .00 \times 0.75=
$$

\section{COSTO DE PRODUCCIÓN (preliminar) $36 \mathbf{6 5 0 0}$}

Base del tiempo. Este método tiende a ser más equitativo, principalmente porque los gastos tienen una relación más directa con el factor tiempo que con el factor precio de la Mano de Obra Directa. Consiste en dividir los GI del mes o del ejercicio anterior entre las horas de trabajo directas del mismo período; el resultado es una cuota por hora que se multiplica por el número de horas empleadas en cada Orden de Servicio, obteniéndose el importe del GI predeterminado, que luego se suma a los costos directos reales para obtener el costo de producción; ejemplo: Adoptando los datos del ejemplo anterior, se obtendría:

Coeficiente GIS $=\frac{\mathrm{S} / .2400000}{500000 \mathrm{H}-\mathrm{H}}=\mathrm{S} / .4 .80$

Las 30000 horas de trabajo directo empleados en la OS \# 001, se multiplica por S/. 4.80 siendo el resultado:

$\begin{array}{lr}\text { - Materiales } & \text { S/. } 100000 \\ \text { - Mano de obra (30 000 H-H) } & 150000 \\ \text { - Gastos indirectos de servicios: } & \\ \qquad 0000 \mathrm{H}-\mathrm{H} \times \mathrm{S} / .4 .80= & 144000 \\ \text { COSTO DE PRODUCCIÓN (preliminar) } & \mathbf{3 2 5 0 0 0}\end{array}$

\section{DETERMINACIÓN DEL COSTO DE PRODUCCIÓN: ANTES O DESPUÉS DEL CIERRE DE LIBROS}

El sistema de costos se hace tangible a través de los formatos de hojas de hojas de costo, adaptables a los procesos operativos o de producción de las empresas, lo que se entrelaza y complementa con las cuentas que reúnen el costo de producción. Las cuentas analíticas de explotación agrupan a las que representa los gastos incurridos por la empresa en la fabricación de bienes o prestación de servicios, que son inherentes a sus actividades y aplicables al ejercicio.
La forma específica de estructurar las cuentas de costos tiene relación con la rapidez y/o exactitud con que la gerencia solicita a contabilidad la información de los costos y además, el plazo breve o prolongado en que se cierra la contabilidad del mes, por consiguiente puede adoptar dos formas:

a. El área de contabilidad tiene plazo amplio para determinar el costo de producción, después del cierre de libros del mes.

Si hubiera tiempo suficiente, se determina el costo real por cada clase de servicio u órdenes de producción, proveniente de la acumulación de las sumas registradas en la cuenta principal que reúne los costos del mes, como: 92 Costo de producción o 93 Centros de costo. Estas cuentas comprende subcuentas que identifique a cada elemento del costo, como por ejemplo: 1 Materiales, 2 Mano de obra, y 9 Gastos indirectos de servicios.

La forma de utilizar las cuentas de costos depende principalmente, de la rapidez o frecuencia con que la gerencia requiera conocer el costo específico de cada servicio prestado, proceso u orden de fabricación. Cuando se determine el costo de cada uno (a través de las hojas de costos), por los recursos utilizados o invertidos (previo cálculo de prorrateo) del costo acumulado real extraído de la cuenta 92 o 93 al cierre del mes. Según el estado de la producción y costo asignado, se procede a cargar a la subcuenta 215 Existencia de servicios terminados y/o 235 Existencias de servicios en proceso y abono a 711 Variación de productos terminados y/o 713 Variación de productos en proceso, respectivamente, a valor real.

$\mathrm{Si}$, posteriormente, los servicios o productos terminados se hubieran facturado, entonces, en aplicación de los PCGA de Equidad y Período, se procede a registrar como gasto real, con cargo a la cuenta 692 Productos terminados y abono a 215 Existencia de servicios terminados.

Respecto a los productos en proceso registrado al cierre del mes, al inicio del siguiente mes y en el entendido que los productos en elaboración o servicios continúan prestándose, se ha de formular el siguiente asiento contable, por la misma suma del mes anterior, así en el caso de una clínica: 
713 Variación de productos en proceso

235 a Existencia de servicios en proceso

931 Gerencia médica- Materiales

932 Gerencia médica- Mano de obra

939 Gerencia médica- Gastos indirectos de servicios

791 a Cargas imputables a cuentas de costos y gastos

Los recursos adicionales del mes aplicados a la orden de servicio en proceso del mes anterior, se contabiliza en la cuenta 93 Centro de costos (o 92 Costo de producción), y cuentas regulares establecidas para cada elemento del costo hasta la conclusión del servicio, repitiéndose el proceso y registro señalado en los párrafos precedentes.

b. El área de contabilidad tiene plazo reducido para determinar el costo de producción y/o requiere determinar el costo de cada servicio antes del cierre contable del mes.

Lo expresado significa que al concluir la fabricación de los productos o prestación del servicio al cliente, previa conformidad se emite la factura y a las 48 horas la gerencia solicita al Contador conocer el costo a valor estimado a efectos de evaluar el comportamiento de los costos y el nivel de rentabilidad, y efectuar los ajustes al valor de venta a futuros clientes, por productos servicios iguales o semejantes, dado la necesidad de mantenerse dentro de los niveles de competitivos de mercado. Posteriormente, se informará del costo real y desviaciones generadas.

Registrar en la contabilidad los costos estimados o estándar conjuntamente con los costos reales, puede adoptar varias formas, como emplear la cuenta 91 Costos por distribuir, en la que se registraría los documentos y provisiones contables (a valor real), no identificados con los productos elaborados o servicios prestados, además de incorporar las subcuentas: 911 Materiales (no aplicable en caso que los suministros se registren a valor real), 912 Mano de obra y 919 Gastos indirectos de servicios. Los costos asignados a valor estimado a cada orden de servicio, se contabiliza con cargo a la cuenta 92 Costo de producción y abono a 91 Costos por distribuir, este último para descargar el costo real acumulado; o en lugar de las cuentas señaladas registrar directamente a
$\operatorname{XXXXXX}$

$\operatorname{XXXXXX}$

$\operatorname{xxxxxx}$

$\operatorname{xxxxxx}$

$\operatorname{XXXXXX}$

valor real en las cuentas de existencias 21 Productos terminados o 23 productos en proceso.

En la situación expuesta, determinar los costos de producción es más laborioso e implica sobrecargar los tratamientos contables, siempre que el contador determine la necesidad de incorporar a la contabilidad, los recursos utilizados valorizados a costos estimados en cada orden de producción o servicios, y según concluyan, se cierran transitoriamente los formatos, cuyo resultado se informa a gerencia, luego al cierre del mes se determina el costo real, procediendo a prorratear las diferencias de costos por cada elemento, y asignar estos ajustes a cada orden de servicio, para cuantificar el costo real. Determinado el costo real este recién se registra en la contabilidad.

Es evidente que establecer los costos de producción en cualquier día del mes, antes de efectuar el cierre contable del mes, el común de los profesionales contables lo considera impracticable. Sin embargo, si es posible establecer el costo de producción, pero transitoriamente a costo estimado o estándar, y al concluir y cerrar el mes, convertirlos a valor real, el que puede adoptar los métodos de contabilización que se describen a continuación:

b.1. Acumular los costos reales no identificados con la prestación de servicios, en la cuenta 91 Costos por distribuir y los costos asignados a cada producto o servicio en la cuenta 92 Costo de producción.

En este método los documentos y provisiones contables que inciden en los costos de producción, se registran además de las cuentas del elemento 6 gastos por naturaleza, en la cuenta 91 Costos por distribuir, y los costos asignados a cada orden de servicio por los recursos utilizados a valor estimado, en la siguiente forma: 
92-2 Costo de producción- Mano de obra

$\operatorname{xxxxxx}$

92-2 Costo de producción- Gastos indirectos de servicios

$\operatorname{xxxxxx}$

91-2 a Costos por distribuir- Mano de obra

$\operatorname{xxxxxx}$

91-9 a Costos por distribuir- Gastos indirectos de servicios

$\operatorname{xxxxxx}$

El asiento contable no incluye al elemento "Materiales", porque generalmente estos son retirados del Almacén, para determinada orden de producción o servicios y se registra a costo real, en la siguiente forma:

61-3 Variación de existencias- Materiales aux., suministros y rep. $\quad$ xxxxxx

25-1 a Materiales auxiliares

$\operatorname{xxxxxx}$

92-1 Costo de producción- Materiales

$\operatorname{xxxxxx}$

79-1 a Cargas imputables a cuentas de costos y gastos

$\operatorname{xxxxxx}$

Posteriormente, previo cálculo, se procede a ajustar los costos estimados de todas las órdenes de producción, a efectos de convertirlos a valor real, y entre otros, cumplir con lo establecido en el párrafo final del artículo $35^{\circ}$ del reglamento de la LIR aprobado con D.S. $\mathrm{N}^{\circ} 122-94-\mathrm{EF}$, el que entre otros enuncia: “ ... los deudores tributarios podrán llevar un Sistema de Costos Estándar que se adapte a su giro, pero al formular cualquier balance para efectos de Impuesto, deberán necesariamente valorar sus existencias al costo real ..."

\section{COSTOS POR DISTRIBUIR}

Costos reales acumulados, pendientes de asignar a los servicios.

Costo estimado, asignado a un servicio específico.

\section{COSTO DE PRODUCCIÓN}

Costo estimado, asignado a un servicio específico

El saldo de la cuenta 91 Costos por distribuir, constituye la diferencia entre el costo real (DB) y el costo estimado (CR), a de generar un saldo deudor o acreedor, es deudor si el costo real (global, no identificado por servicio o cliente) es mayor que el estimado (identificado por cada servicio), lo que significa que por el saldo deudor se cierra la cuenta formulando el asiento de diario: 92/91. Si el costo real es menor que el costo estimado, el saldo es acreedor, luego se traslada el saldo a la cuenta 92 Costo de producción, formulando el A/D: 91/92. A fin de mes en cualquie- ra de las dos posibilidades, la cuenta 92 ha de revelar el costo real, pero convertido en un costo analítico y detallado por cada orden de servicio, sustentado en las hojas de costo respectivas, entrelazadas y conciliadas con las cuentas de control de costos del libro mayor. Luego se carga a la subcuenta 215 Existencia de servicios terminados y/o 235 Existencias de servicios en proceso y abono a 711 Variación de productos terminados y/o 713 Variación de productos en proceso, respectivamente, a valor real. La cuenta 91 Costos por distribuir queda saldada. 
Una alternativa de contabilización es que en lugar de emplear la cuenta 91 Costos por distribuir, se aplica la cuenta 93 Centros de costos, para registrar los costos reales y por los recursos utilizados y valorizados a costo estimado, se registra:

92 Costo de producción $\quad \mathrm{xxxxxx}$

93 a Centros de costo

$\operatorname{xxxxxx}$

El tratamiento contable posterior es igual al aplicado a la cuenta 91 Costos por distribuir.

b.2. Acumular los costos reales no identificados con la prestación de servicios en la cuenta 92 Costo de producción y los costos asignados (estimados) a cada servicio en las cuentas de existencias 21 Productos terminados o 23 Productos en proceso, a valor estimado, y luego se adiciona o se resta los ajustes de costo en las cuentas de existencias.

En este método el costo real se mantiene en la cuenta 92 Costo de producción. Se contabiliza a costo estimado las órdenes de servicio concluidas o en proceso, es debitar a la cuenta 21 Productos terminados o 23 Productos en proceso, con abono a la cuenta 71 Variación de la producción almacenada, luego formular el ajuste de costos. Si el costo real acumulado en la cuenta 92 costo de producción es mayor que el costo asignado, entonces se formula el A/D: 21/71 y/o 23/71, pero si el costo real acumulado es menor que el costo asignado, entonces el A/D será: $71 / 21$ o $71 / 23$.

En las empresas que sea apropiado emplear la cuenta 93 Centros de costos, en lugar de la cuenta 92 Costo de producción, tendrá el mismo tratamiento contable antes descrito.

b.3. Acumular los costos reales no identificados con la prestación de servicios en la cuenta 92 Costo de producción y los costos asignados (estimados) a cada servicio no se contabilizan hasta que sean ajustados y cuantificado el costo real, en las cuentas de existencias 21 Productos terminados o 23 Productos en proceso.

Este método restringe la sobrecarga de las registraciones contables, dado que si bien en los formatos de órdenes de servicios se registran los recursos utilizados a costo estimado, y con estos valores se informa con carácter preliminar a la gerencia, no se contabilizan. Posteriormente, se incorpora los ajustes de costos a los formatos, sea incrementando o disminuyendo el costo estimado, para llegar a la suma de costo real, las que deben ser coincidentes con el saldo de la cuenta 92 Costo de producción, recién entonces las existencias se registran a costo real, en las cuentas 215 Existencias de servicios terminados y/o 235 Existencia de servicios en proceso, con abono a las cuentas 711 Variación de productos terminados o 13 Variación de productos en proceso.

En las empresas que sea apropiado emplear la cuenta 93 Centros de costos, en lugar de la cuenta 92 Costo de producción, tendrá el mismo tratamiento contable que la primera cuenta.

\section{NORMAS DEL IMPUESTO A LA RENTA QUE REGULAN LA CONTABILIDAD DE COSTOS}

El TUO de la Ley del Impuesto a la Renta, aprobado con D.S. $N^{\circ} 179-2004-E F$ y el reglamento de la LIR aprobado con D.S. N 122-94-EF regula el sistema de contabilidad de costos, para efectos tributarios; así entre otros, tenemos:

El numeral 2 del artículo $20^{\circ}$ de la LIR enuncia que el "Costo de producción o construcción, es el costo incurrido en la producción o construcción del bien, el cual comprende los materiales directos utilizados, la mano de obra directa y los costos indirectos de fabricación o construcción”. La norma reconoce la participación de los tres elementos del costo de producción, el que puede estar referido a bienes o servicios.

El artículo $35^{\circ}$ del reglamento de la LIR Inventarios y Contabilidad de Costos, enuncia:

"Los deudores tributarios deberán llevar sus inventarios y contabilizar sus costos de acuerdo a las siguientes normas:

a) Cuando sus ingresos brutos anuales durante el ejercicio precedente hayan sido mayores a 1500 UIT del ejercicio en curso, deberán llevar un sistema de contabilidad de costos, cuya información deberá ser registrada en los siguientes registros: Registro de Costos, Registro de Inventario Permanente en Unidades Físicas y Registro de Inventario Permanente Valorizado. 
b) Cuando sus ingresos brutos anuales durante el ejercicio precedente hayan sido mayores o iguales a 500 UIT y menores o iguales a 1500 UIT del ejercicio en curso, solo deberán llevar un Registro de Inventario Permanente en Unidades Físicas.

c) Cuando sus ingresos brutos anuales durante el ejercicio precedente hayan sido inferiores a 500 UIT del ejercicio en curso, solo deberán realizar inventarios físicos de sus existencias al final del ejercicio.

d) Tratándose de los deudores tributarios comprendidos en los incisos precedentes a) y b), adicionalmente deberán realizar, por lo menos, un inventario físico de sus existencias en cada ejercicio.

e) Deberán contabilizar en un Registro de Costos, en cuentas separadas, los elementos constitutivos del costo de producción por cada etapa del proceso productivo. Dichos elementos son los comprendidos en la Norma Internacional de Contabilidad correspondiente, tales como: materiales directos, mano de obra directa y gastos de producción indirectos.

f) Aquellos que deben llevar un sistema de contabilidad de costos basado en registros de inventario permanente en unidades físicos o valorizados o los que sin estar obligados opten por llevarlo regularmente, podrán deducir pérdidas por faltantes de inventario, en cualquier fecha dentro del ejercicio, siempre que los inventarios físicos y su valorización hayan sido aprobados por los responsables de su ejecución y además, cumplan con lo dispuesto en el segundo párrafo del inciso c) del artículo $21^{\circ}$ del Reglamento.

g) No podrán variar el método de valuación de existencias sin autorización de la SUNAT y surtirá efecto a partir del ejercicio siguiente a aquél en que se otorgue la aprobación, previa realización de los ajustes que dicha entidad determine.

h) La SUNAT mediante Resolución de Superintendencia podrá:

- Establecer los requisitos, características, contenido, forma y condiciones en que deberá llevarse los registros establecidos en el presente artículo.

- Eximir a los deudores tributarios comprendidos en el inciso a) del presente artículo, de llevar el Registro de Inventarios Permanentes en Unidades Físicas.

- Establecer los procedimientos a seguir para la ejecución de la toma de inventarios físicos en armonía con las normas de contabilidad referidas a tales procedimientos.

En todos los casos, en que los deudores tributarios practiquen inventarios físicos de sus existencias, los resultados de dichos inventarios deberán ser refrendados por el Contador o persona responsable de su ejecución y aprobados por el representante legal.

A fin de mostrar el costo real, los deudores tributarios podrán llevar un Sistema de Costos Estándar que se adapte a su giro, pero al formular cualquier balance para efectos de Impuesto, deberán necesariamente valorar sus existencias al costo real. Los deudores tributarios deberán proporcionar el informe y los estudios técnicos necesarios que sustenten la aplicación del sistema antes referido, cuando sea requerido por la SUNAT".

Respecto al párrafo precedente, cabe precisar que el sistema de costos estándar, forma parte de los denominados costos predeterminados, conjuntamente con los denominados costos estimados, teniendo como diferencia principal que el primero son costos calculados con anticipación; en cambio el segundo, es el costo que debería ser, fijado técnicamente.

Por lo expuesto, para que un sistema de costos "funcione", es necesario recurrir transitoriamente a la técnica de costo estimado o proyectado y luego, formular los ajustes por diferencias de costeo para obtener los costos reales, lo que debe quedar evidenciado en los papeles de trabajo.

\section{CONCLUSIONES}

1. En las MYPES frecuentemente no existen las condiciones apropiadas para diseñar e implementar un sistema de costos que permita identificar y cuantificar la participación de los tres elementos del costo de producción: Materia prima, Mano de Obra Directa y Gastos Indirectos de Producción.

2. Si los Directivos de las MYPES necesitan (es indispensable) determinar el costo de producción, pero

Vol. 22(41) 2014 | QVIPURAMAYOC / 133 
para ello no están dispuestos a invertir en recursos operativos y de personal, entonces el Contador Público (de aceptar el reto) deberá formular el sistema de costos en función a la organización y condiciones operativas existentes en la empresa, recurriendo a métodos $y$ procedimientos no convencionales, para lograr alcanzar los objetivos previstos.

\section{RECOMENDACIONES}

1. El Contador Público durante el proceso de formación profesional, iniciado en los estudios de pregrado universitario, ha sido formado en un "modelo ideal", en que todo sistema se implementa en condiciones óptimas, pero en la realidad, es lo que menos abunda. Por consiguiente, tiene dos opciones: rechaza asumir la responsabilidad de formular e implementar un sistema de costos, en condiciones óptimas o acepta la responsabilidad para operar en condiciones no adecuadas y gradualmente en base al óptimo resultado, contribuir al cambio de mentalidad en las Pymes, con el objetivo de organizary disciplinar la organización a efectos de implementar un sistema regular de costos.

\section{REFERENCIAS BIBLIOGRÁFICAS}

1. Garrison, R., Noreen E.y Brewer, P. (2007) Contabilidad Administrativa. México: Mc Graw-Hill

2. Hansen R. y Mowen M. (2009) Contabilidad de Costos, Contabilidad y Control. México: Cengage Learning Editores, S.A.

3. Polimeni, R., Fabozzi, F., Adelberg, A. y Kole, M. (2010) Contabilidad de Costos, Conceptos y Aplicaciones para la Toma de Decisiones Gerenciales. Colombia: McGraw-Hill. 